\title{
MTA1 is an indicator of prognosis in Chinese patients with lung adenocarcinoma
}

\author{
Yue Deng" ${ }^{\#}$ Jian Yu" \\ Department of Gerontology, The First People's Hospital of Changzhou, Third Affiliated Hospital of Soochow University, Changzhou 213003, China \\ Contributions: (I) Conception and design: All authors; (II) Administrative support: None; (III) Provision of study materials or patients: All authors; \\ (IV) Collection and assembly of data: All authors; (V) Data analysis and interpretation: All authors; (VI) Manuscript writing: All authors; (VII) Final \\ approval of manuscript: All authors. \\ "These authors contributed equally to this work. \\ Correspondence to: Jian Yu. Department of Gerontology, The First People's Hospital of Changzhou, Third Affiliated Hospital of Soochow University, \\ 185\# Juqian Street, Changzhou 213003, China. Email: submanuscript2013@gmail.com.
}

Background: The predictive role of metastatic tumor antigen 1 (MTA1) expression in lung cancer (LC) is still uncertain. This meta-analysis was performed to clarify the relationship of MTA1 protein expression with the prognosis of LC.

Methods: The PubMed and CNKI databases were searched for clinical studies demonstrating the expression of MTA1 in LC for further review.

Results: A total of ten studies comprising 982 patients were included in the meta-analysis according to the predefined selection criteria. Our results indicated that MTA1 overexpression had a significant impact on progression-free survival (PFS) (HR 2.16; 95\% CI, 1.62-2.89) and OS (HR 2.38; 95\% CI, 1.78-3.19). Heterogeneity was observed $\left(\mathrm{I}^{2}=34.6 \%\right)$. When the analyses were restricted to adenocarcinoma cases, high expression of MTA1 also predicted poor prognosis (HR 2.14; 95\% CI, 1.48-3.08). However, no statistically significant heterogeneity was observed among the studies $\left(\mathrm{I}^{2}=4.1 \%\right)$.

Conclusions: A high level of MTA1 expression predicts poor survival in Chinese patients with lung adenocarcinoma.

Keywords: Metastatic tumor antigen 1 (MTA1); prognosis; lung cancer (LC); adenocarcinoma; meta-analysis

Submitted Oct 21, 2019. Accepted for publication Jan 07, 2020.

doi: $10.21037 /$ tcr.2020.01.68

View this article at: http://dx.doi.org/10.21037/tcr.2020.01.68

\section{Introduction}

Lung cancer (LC) is one of the most common human malignant diseases and the leading cause of cancer-related death globally (1). A further understanding of the mechanisms underlying the pathogenesis and progression of LC would promote the development of novel prognostic markers and therapeutic targets. The selection of appropriate treatment for each individual patient is determined by several factors, including the assessment of prognosis. Epithelial-tomesenchymal transition (EMT) is a key step in the progression of tumor cell metastasis. Various types of metastasis-associated cancer genes and proteins have been investigated extensively. Metastatic tumor antigen 1 (MTA1), a core component of the nucleosome remodeling and deacetylase (NuRD) complex, appears to correlate with human cancer progression and metastasis $(2,3)$. High levels of MTA1 expression have been shown to be involved in migration, invasion, metastasis, early recurrence, and poor prognosis, suggesting that MTA1 is a molecular target for future anticancer therapeutics. Therefore, MTA1 may be a new indicator of cancer prognosis. A previous meta-analysis published in 2014 found that overexpression of MTA1 in non-small cell lung cancer (NSCLC) patients might be a poor prognostic factor for survival (4). Only 4 studies were included in this meta-analysis (5-8). However, the fact that the significant associations were based on a limited number of studies may reduce the statistical power. 
Table 1 Characteristic of the studies for meta-analysis for MTA1 on NSCLCs

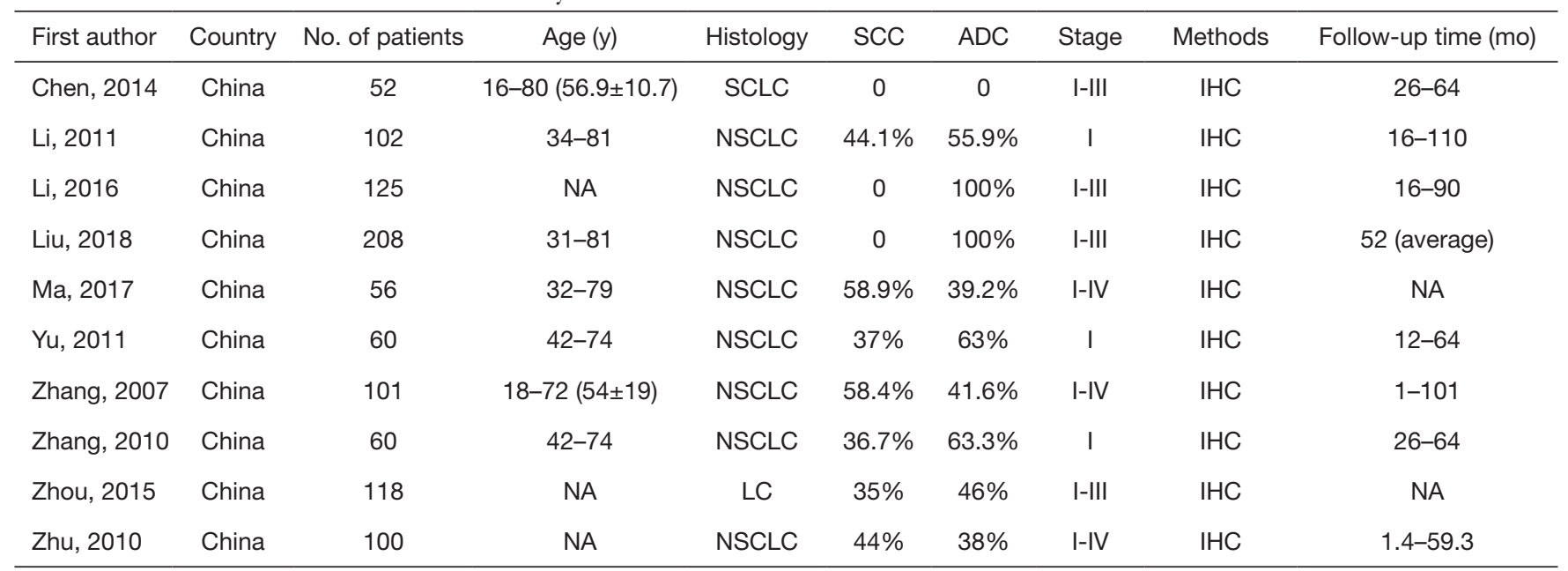

NSCLC, non-small cell lung cancer; SCC, squamous cell carcinoma; ADC, adenocarcinoma; NA, not available.

The role of MTA1 expression in lung cancer patients remains uncertain. Therefore, to derive a more precise evaluation of the association, we performed this meta-analysis.

\section{Methods}

\section{Publication search}

The PubMed and CNKI databases were searched for clinical studies demonstrating the expression of MTA1 in LC for further review using the keywords "MTA1", "lung cancer", and "lung carcinoma". An early date limit of August 31, 2019, was used. Additional publications were identified from review articles and from the references cited in the previously found articles. Studies included in the meta-analysis were in any language, studied human subjects, and were primary studies. Only published full-text articles were reviewed and assessed for relevant content. When several papers from the same study had been published, only the most complete or most recent paper was used.

\section{Data extraction and statistical analysis}

Study characteristics, such as first authors, year of publication, region/country, number of cases, and the cut-off values of MTA1, were noted. The hazard ratio (HR) was used as the outcome measure for time-to-event data. When HRs were not captured in a study, they were calculated according to the data extracted from the Kaplan-Meier curves (9). To account for the heterogeneity, a random effects model was used to pool the data because of the wide confidence intervals (CIs).
Cochran's Q statistic was used to assess heterogeneity. Funnel plots and Egger's linear regression test were used to inspect potential publication bias. Subgroup analyses were performed to eliminate heterogeneity by using the following criteria: (I) NSCLC; (II) stage I; (III) adenocarcinoma; and (IV) squamous cell carcinoma rate $>50 \%$. Stata 10.0 was used to perform statistical analysis.

\section{Results}

\section{Study characteristics}

Our search strategy identified a total of 251 articles. After reading the titles and abstracts, 27 articles were selected for full reading. Finally, ten studies published between 2007 and 2018 comprising 982 patients were included in the study (5-8,10-15). The characteristics of the studies are shown in Table 1. In the 10 studies, the sample sizes ranged from 52 to 208. Four studies provided progression-free survival (PFS) outcome data $(5,12,13,15)$. Immunohistochemistry (IHC) was used to confirm the expression of MTA1. Different expression values of MTA1 were used as cut-off points. Few of MTA1 expression was positive in adjacent nonneoplastic lung tissues (OR 18.24; 95\% CI, 8.86-2.37.54, compared with tumoral tissues).

\section{Meta-analysis results}

The outcome of meta-analysis is shown in Figure 1. The protein expression levels of MTA1 were significantly associated with the PFS (HR 2.16; 95\% CI, 1.62-2.89) and OS (HR 2.38; 95\% CI, 1.78-3.19, Figure 2). Heterogeneity 


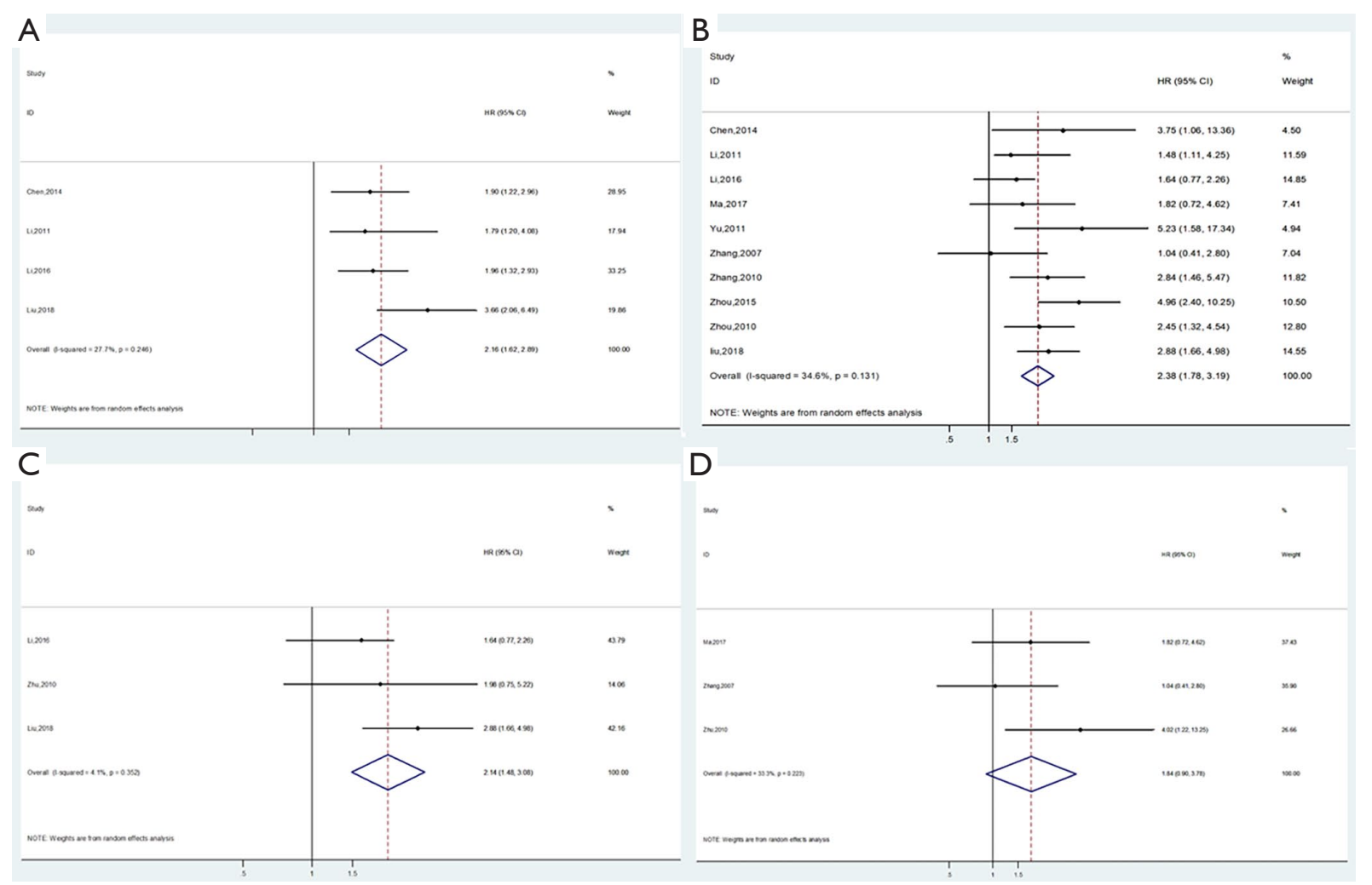

Figure 1 Forest plot. (A) PFS; (B) OS; (C) adenocarcinoma; (D) squamous cell carcinoma. PFS, progression-free survival; OS, overall survival.

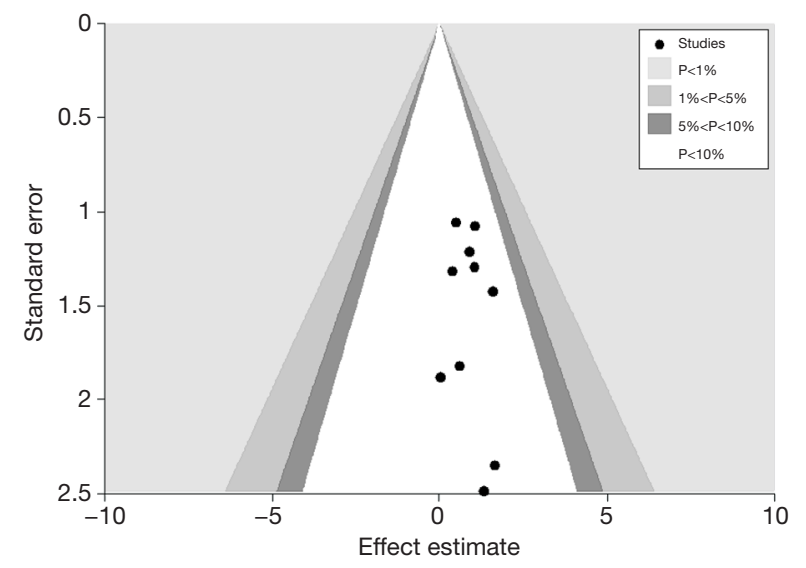

Figure 2 Funnel plot for publication bias.

was observed $\left(\mathrm{I}^{2}=34.6 \%\right)$. The subgroup analysis was performed according to the protocol described in the methods section, and the outcome is shown in Table 2. When the analyses were restricted to adenocarcinoma cases, high expression of MTA1 also predicted poor prognosis (HR 2.14; $95 \%$ CI, 1.48-3.08). No significant heterogeneity was observed among the studies $\left(\mathrm{I}^{2}=4.1 \%\right)$.

\section{Publication bias}

Publication bias was assessed using a funnel plot and Egger's test. No obvious publication bias was observed (Egger's test, $\mathrm{P}=0.421$, Figure 3).

\section{Discussion}

Exploring the association between novel biomarkers and the prognosis of LC is important for improving the clinical prognosis of LC. MTA1 is known to increase the migration, invasion and metastasis of cancer cells $(16,17)$. However, the mechanism by which MTA1 affects tumor growth and metastasis remains unclear. MTA1 might promote tumor growth and metastasis via the AKT/GSK3 $\beta / \beta$-catenin signaling pathway (10). MTA1 has been reported to mediate PTEN acetylation and indirectly activate AKT (18).

Some studies indicated that MTA1 was a predictor for clinical outcome $(5,8,14)$, but others did not replicate this association $(6,10,13)$. At present, no consensus has been reached regarding whether MTA1 could be a predictive marker in patients with LC. To derive a more precise estimation of the prognostic role of MTA1 expression, we 
Table 2 Subgroup analyses for OS according to study characteristics

\begin{tabular}{lccr}
\hline \multirow{2}{*}{ Variables } & $\mathrm{HR}$ & \multicolumn{2}{c}{ Heterogeneity } \\
\cline { 2 - 4 } & Random effects $(95 \% \mathrm{Cl})$ & $\mathrm{I}^{2}(\%)$ & $\mathrm{P}$ value Q test \\
\hline Total & $2.38(1.78-3.19)$ & 17.9 & 0.131 \\
NSCLC & $1.21(1.62-2.80)$ & 48.1 & 0.288 \\
Stages I & $2.48(1.31-4.71)$ & 4.1 & 0.146 \\
Adenocarcinoma & $2.14(1.48-3.08)$ & 33.3 & 0.352 \\
Squamous cell carcinoma & $1.84(0.90-3.78)$ & 0.223 \\
\hline
\end{tabular}

NSCLC, non-small cell lung cancer; HR, hazard ratio; OS, overall survival; Cl, confidence interval.

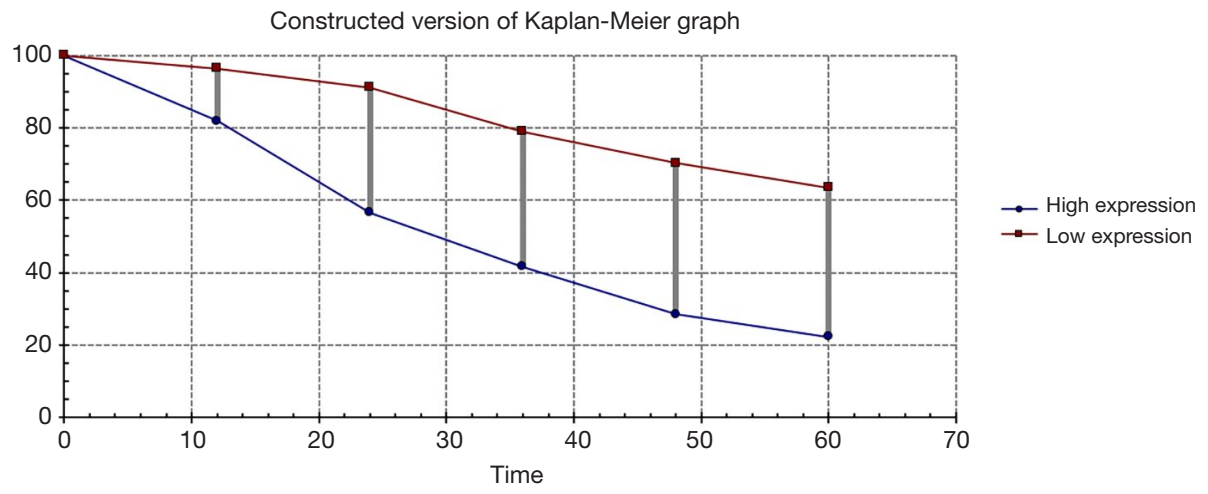

Figure 3 Kaplan-Meier survival curves for MTA1 expression LC patients according to MTA1 expression. LC, lung cancer; MTA1, metastatic tumor antigen 1 .

performed an updated meta-analysis of all studies. Only one study provided the survival data of squamous cell carcinoma (7), so we used a squamous cell carcinoma rate of $50 \%$ as a cutoff value to complete the subgroup analyses. When we subgrouped the studies according to histology, we found that MTA1 was not a prognostic marker for squamous cell carcinoma. However, high expression of MTA1 was associated with an adverse outcome in patients with adenocarcinoma. There were only 3 studies based on adenocarcinoma that obtained positive results $(7,13,15)$. All studies selected in this study were from China. The results were similar to previous studies and suggest that these studies were representative. Our results thus far suggest that MTA1 might be a potential target for anti-adenocarcinoma therapy. However, further research is needed to clarify the association.

The final results from a meta-analysis should be interpreted extremely carefully, particularly when the number of studies is small and the heterogeneity is large. The previously published meta-analysis had only four studies to evaluate the influence of the expression of MTA1 on the prognosis of NSCLC (4), and significant heterogeneity was observed $(\mathrm{P}=0.04)$. Subgroup analyses were performed to eliminate heterogeneity in our study. In adenocarcinoma cases, no statistically significant heterogeneity was observed, which might reveal that the clinical outcome of LC may differ by histology.

Some limitations of this study should be taken into consideration. First, among the studies included in this meta-analysis, all were conducted in China. Thus, data representing other ethnicities were not sufficient. Second, the inherent biases of patients may lead to spurious correlations. Third, the small sample size may lack sufficient power to identify real interactions. Fourth, subgroup analysis according to MTA1 IHC positivity was not conducted because of the different methods used to determine the staining intensity and proportion of positively stained cancer cells in the various studies. Finally, subgroup analyses based on age, sex, and other factors (such as smoking status consumption and diabetes) were not performed in this study because relevant data were not available in the primary articles.

In conclusion, a high level of MTA1 expression predicts poor survival in Chinese patients with lung adenocarcinoma. 


\section{Acknowledgments}

Funding: None.

\section{Footnote}

Conflicts of Interest: Both authors have completed the ICMJE uniform disclosure form (available at http://dx.doi. org/10.21037/tcr.2020.01.68). The authors have no conflicts of interest to declare.

Ethical Statement: The authors are accountable for all aspects of the work in ensuring that questions related to the accuracy or integrity of any part of the work are appropriately investigated and resolved.

Open Access Statement: This is an Open Access article distributed in accordance with the Creative Commons Attribution-NonCommercial-NoDerivs 4.0 International License (CC BY-NC-ND 4.0), which permits the noncommercial replication and distribution of the article with the strict proviso that no changes or edits are made and the original work is properly cited (including links to both the formal publication through the relevant DOI and the license). See: https://creativecommons.org/licenses/by-nc-nd/4.0/.

\section{References}

1. Chen $\mathrm{W}$, Zheng R, Baade PD, et al. Cancer statistics in China, 2015. CA Cancer J Clin 2016;66:115-32.

2. Kumar R, Wang RA. Structure, expression and functions of MTA genes. Gene 2016;582:112-21.

3. Kaur E, Gupta S, Dutt S. Clinical implications of MTA proteins in human cancer. Cancer Metastasis Rev 2014;33:1017-24.

4. Luo H, Li H, Yao N, et al. Metastasis-associated protein 1 as a new prognostic marker for solid tumors: a metaanalysis of cohort studies. Tumour Biol 2014;35:5823-32.

5. Li SH, Tian H, Yue WM, et al. Overexpression of metastasis-associated protein 1 is significantly correlated with tumor angiogenesis and poor survival in patients with early-stage non-small cell lung cancer. Ann Surg Oncol 2011;18:2048-56.

6. Zhang N, Zhang Q, Wang E, et al. Relationship between metastasis-associated protein 1 expression and metastasis and prognosis in non-small cell lung cancer. Zhongguo Fei Ai Za Zhi 2007;10:279-83.

7. Zhu X, Guo Y, Li X, et al. Metastasis-associated protein 1 nuclear expression is associated with tumor progression and clinical outcome in patients with non-small cell lung cancer. J Thorac Oncol 2010;5:1159-66.

8. Yu Y, Wang Z, Zhang MY, et al. Relation between prognosis and expression of metastasis-associated protein 1 in stage I non-small cell lung cancer. Interact Cardiovasc Thorac Surg 2011;12:166-9.

9. Tierney JF, Stewart LA, Ghersi D, et al. Practical methods for incorporating summary time-to-event data into metaanalysis. Trials 2007;8:16.

10. Ma K, Fan Y, Dong X, et al. MTA1 promotes epithelial to mesenchymal transition and metastasis in non-small-cell lung cancer. Oncotarget 2017;8:38825-40.

11. Zhou N, Wang H, Liu H, et al. MTA1-upregulated EpCAM is associated with metastatic behaviors and poor prognosis in lung cancer. J Exp Clin Cancer Res 2015;34:157.

12. Chen YT, Xu YC, Tao LL, et al. Expression of MTA1 protein in small cell lung cancer and its correlation with chemotherapy sensitivity and prognosis. Lin Chuang Zhong Liu Xue Za Zhi 2014;19:102-6.

13. Li S, Tian H, Yue W, et al. Clinicopathological and prognostic significance of metastasis-associated protein 1 expression and its correlation with angiogenesis in lung invasive adenocarcinomas, based on the 2011 IASLC/ATS/ ERS classification. Oncol Lett 2016;11:224-30.

14. Zhang H, Zhang YM, Wang Z, et al. Expression of metastasis-associated gene 1(MTAI) protein and its association with the prognosis of stage I non-small cell lung cancer. Zhong Liu 2010;30:881-5.

15. Liu Y, Chen D, Qiu X, et al. Relationship between MTA1 and spread through air space and their joint influence on prognosis of patients with stage I-III lung adenocarcinoma. Lung Cancer 2018;124:211-8.

16. Mahoney MG, Simpson A, Jost M, et al. Metastasisassociated protein (MTA)1 enhances migration, invasion, and anchorage-independent survival of immortalized human keratinocytes. Oncogene 2002;21:2161-70.

17. Hofer MD, Menke A, Genze F, et al. Expression of MTA1 promotes motility and invasiveness of PANC-1 pancreatic carcinoma cells. Br J Cancer 2004;90:455-62.

18. Dhar S, Kumar A, Li K, et al. Resveratrol regulates PTEN/Akt pathway through inhibition of MTA1/HDAC unit of the NuRD complex in prostate cancer. Biochim Biophys Acta 2015;1853:265-75.

Cite this article as: Deng Y, Yu J. MTA1 is an indicator of prognosis in Chinese patients with lung adenocarcinoma. Transl Cancer Res 2020;9(3):1625-1629. doi: 10.21037/tcr.2020.01.68 